\title{
CONTINUED FUNCTION EXPANSIONS OF REAL NUMBERS ${ }^{1}$
}

\author{
B. K. SWARTZ AND B. WENDROFF
}

1. Introduction. We present a theory of continued function expansions of numbers which contains the generalized continued fractions of B. H. Bissinger [1] and the generalized decimal representations of C. J. Everett [2]. The latter used the following algorithm for representing numbers as sequences of integers: for any $\gamma \geqq 0$ let $\gamma_{0}=\gamma$, $\gamma_{n+1}=f^{-1}\left(\gamma_{n}-a_{n}\right)$, where $a_{n}=\left[\gamma_{n}\right]$ and $f$ is strictly increasing and continuous from $[0, p]$ onto $[0,1], p$ an integer. We generalize this, in particular, by admitting a wider class of functions than those of the form $f^{-1}(x-n)$. O. W. Rechard [3] gave a necessary and sufficient condition that the correspondence between numbers and sequences resulting from Everett's algorithm be 1-1. This condition appears in our theory as a simple functional relation similar to one considered by Schreier and Ulam [4].

2. The algorithm. The correspondence between numbers and sequences which we are going to describe depends on a collection of intervals and on functions defined on those intervals. More precisely

Definition. An algorithm frame, $A$, consists of the following: an interval $R$; a subset $P$ of the integers containing at least two integers; a partition of $R$ into disjoint intervals $I_{n}, n \in P$; a subset $P_{0}$ of $P$ containing at least two integers such that $I=\bigcup_{n \in P_{0}} I_{n}$ is an interval; intervals $M_{n}, n \in P$, homeomorphic to each other such that $M_{n} \subset I_{n}$ and $I_{n}-M_{n}$ consists of at most one point; and an interval $M$ homeomorphic to each $M_{n}$ such that $\mathrm{U}_{n \in P_{0}} M_{n} \subset M \subset I$.

It follows from the above definition that if $\left\{M_{n}, n \in P\right\}$ is part of an algorithm frame then either all the $M_{n}$ are open intervals or all are closed on one end, not necessarily the same, because not all the $I_{n}$ can be closed and the $M_{n}$ are homeomorphic to each other. Also, if any interval is infinite at some end it is taken to be open at that end.

Definition. $A n$ algorithm basis consists of an algorithm frame $A$ and a collection of homeomorphisms $h_{n}, n \in P$, mapping $M_{n}$ onto $M$. We usually identify an algorithm basis by the couple $\left(A, h_{n}\right)$.

Corresponding to any algorithm basis we have the following algorithm for relating points in $R$ to sequences (finite or infinite) of integers:

Presented to the Society, January 29, 1960; received by the editors October 9, 1959.

${ }^{1}$ Work performed under A.E.C. Contract No. W-7405-Eng. 36. 
Let $x_{0} \in R$. $a(0)$ :

$\left\{a(0)\right.$ is determined by the requirement that $\left.x_{0} \in I_{a(0)}.\right\}$

$\left\{\begin{array}{l}\text { If } x_{0} \notin M_{a(0)}, \text { stop, and represent } x_{0} \text { by the sequence of } \\ \text { one element }\{a(0)\}\end{array}\right\}$

$\left\{\begin{array}{l}\text { Since } x_{0} \in M_{a(0)} \text { we can let } x_{1}=h_{a(0)}\left(x_{0}\right) \text {. Then } a(1) \\ \text { is determined by the requirement that } x_{1} \in I_{a(1)} \\ \text { Furthermore, } a(1) \in P_{0} \text { since } x_{1} \in M \subset I .\end{array}\right\}$ $a(1)$ :

$\left.\begin{array}{l}\text { If } x_{1} \notin M_{a(1)} \text {, stop, and represent } x_{0} \text { by the sequence of } \\ \text { two elements }\{a(0), a(1)\} \text {. }\end{array}\right\}$

$a(k)$ :

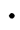

Since $x_{k-1} \in M_{a(k-1)}$ we can let $x_{k}=h_{a(k-1)}\left(x_{k-1}\right)$.

$\left\{\begin{array}{l}\text { Then } a(k) \text { is determined by the requirement that } \\ x_{k} \in I_{a(k)}, \text { and } a(k) \in P_{0} .\end{array}\right.$

$\left\{\right.$ If $x_{k} \notin M_{a(k)}$, stop, and represent $x_{0}$ by $\left.\{a(0), \cdots, a(k)\}.\right\}$

This algorithm contains the expansions considered by Bissinger and Everett. Let $A B$ be the following algorithm basis:

$$
\begin{aligned}
R=[0, \infty), P & =\{0,1,2, \cdots\}, P_{0}=\{1,2, \cdots\}, I_{n}=[n, n+1), \\
M_{n} & =(n, n+1), \quad I=[1, \infty), \quad M=(1, \infty),
\end{aligned}
$$

and let $h_{n}(x)=f^{-1}(x-n)$ for $x \in(n, n+1)$ where $f$ is a continuous strictly decreasing function mapping $[1, \infty)$ onto $(0,1]$. This contains Bissinger's expansions. Everett's expansions come from the algorithm basis $A E$ given by:

$$
\begin{gathered}
R=I=M=[0, p), \quad I_{n}=M_{n}=[n, n+1), \\
P=P_{0}=\{0,1, \cdots, p-1\},
\end{gathered}
$$

and $h_{n}(x)=f^{-1}(x-n), x \in[n, n+1)$ where $f$ is continuous and strictly increasing from $[0, p]$ onto $[0,1]$.

3. 1-1 Correspondence. Given an algorithm basis $\left(A, h_{n}\right)$, the algorithm defines a function $h$ from $R$ into the space $C$ of finite or infinite sequences of integers $c=\{c(0), c(1), \cdots\}$ as follows: let $x$ 
yield $c$ under the algorithm, then $h(x)=c$. Let $E$ be the set of all such functions. In general we will use the convention that if $g \in E$ then the homeomorphisms in its algorithm basis are $g_{n}$.

Definition. Let $\left(A, h_{n}\right),\left(B, g_{n}\right)$ be algorithm bases. The corresponding functions $h$ and $g \in E$ are said to be equivalent, written $h \sim g$, if $A$ and $B$ are identical and if $h_{n}$ has the same sense as $g_{n}$ for each $n$. (By this we mean that if $h_{n}$ is monotonic increasing so is $g_{n}$ and if $h_{n}$ is monotonic decreasing so is $g_{n}$. This is not meant to imply that the sense of $h_{n}$ is independent of $n$.)

Denote by $C(h)$ the range of $h$ for $h \in E$.

The following theorems characterize the equivalent 1-1 functions in $E$ :

Theorem 1. If $h \sim g$ and $h$ is $1-1$ onto $C(h)$ then $C(h) \subset C(g)$.

COROLLARY 1. If $h \sim g$, a finite sequence is in $C(h)$ if and only if it is in $C(g)$.

Corollary 2. If $g$ is $1-1, C(h)=C(g)$.

Notation. A sequence of functions $h g \cdots k$ always means the composite function $h(g(\cdots(k) \cdots))$.

THEOREM 2. Let $g$ be 1-1 from $R$ onto $C(g)$ and let $h$ have the same algorithm frame as $g$. Then $h \sim g$ and $h$ is $1-1$ from $R$ onto $C(g)$ if and only if there exists an increasing homeomorphism $F$ from $R$ onto $R$, which also maps $M_{n}$ onto $M_{n}$ for all $n$, such that $h_{n}^{-1}=F^{-1} g_{n}^{-1} F$.

The following theorems are an application of Theorem 2 to bases $A B$ and $A E$, respectively.

Theorem 3. Let $\left(A, h_{n}\right)$ be an algorithm basis of the form $A B$. Let $h_{n}(x)=\hat{h}^{-1}(x-n)$. Then $h$ is 1-1 if and only if there exists an increasing homeomorphism $F$ mapping $[0, \infty)$ onto itself such that $F(x)=n$ $+F(x-n)$ for $x \in[n, n+1)$ and $\hat{h}^{-1}(\tau)=F^{-1}(1 / F(\tau))$ for all $\tau \in(0,1]$.

Theorem 4. Let $\left(A, h_{n}\right)$ be an algorithm basis of the form $A E$. Let $h_{n}(x)=\hat{h}^{-1}(x-n)$. Then $h$ is 1-1 if and only if there exists an increasing homeomorphism $F$ mapping $[0, p]$ onto itself such that $F(x)=n$ $+F(x-n)$ for $x \in[n, n+1)$ and $\hat{h}^{-1}(\tau)=F^{-1}(p \cdot F(\tau))$ for all $\tau \in[0,1]$.

Rechard's condition is that $h$ is $1-1$ if and only if there exists an increasing homeomorphism $G$ mapping $[0,1]$ onto itself such that $\hat{h}(y)=G^{-1}((n+G(y-n)) / p)$. It is easily verified that this is equivalent to Theorem 4 (given $G$, set $F(y)=n+G(y-n), y \in[n, n+1$ ), and given $F$ set $G(\tau)=F(\tau), \tau \in[0,1])$.

Proof of Theorem 1. 
LEMMA. Let $\left(A, f_{n}\right)$ be any algorithm basis and let $c$ be any infinite sequence $\{c(0), c(1), \cdots\}$ such that $c(0) \in P, c(i) \in P_{0}$ for $i>0$. Let $F_{k}=f_{c(0)}^{-1} \cdots f_{c(k)}^{-1}(M)=f_{c(0)}^{-1} \cdots f_{c(k-1)}^{-1}\left(M_{c(k)}\right)$. Then $f(x)=c$ if and only if $x \in \cap_{0}^{\infty} F_{k}$.

Proof of Lemma. $F_{k}$ consists exactly of those points $y$ which correspond, under $f$, to sequences with at least $k+2$ entries, the first $k+1$ of which are $c(0), \cdots, c(k)$, and the lemma follows immediately from this fact. Proceeding with the theorem, let $h$ be 1-1 onto $C(h), h \sim g$, and let $h(x)=c$. If $c=\{c(0)\}$, then $g(x)=c$. If $c=\{c(0), \cdots, c(k)\}, \quad k>0$, then $x=h_{c(0)}^{-1} \cdots h_{c(k-1)}^{-1}(y)$ where $y \in I_{c(k)}-M_{c(k)}$ (note that in the definition of algorithm frame it was assumed that $I_{n}-M_{n}$ consists of at most one point; the reason for this is apparent, for if there were more than one point $h$ could not be 1-1). Then if $w=g_{c(0)}^{-1} \cdots g_{c(k)}^{-1}(y), g(w)=c$. If $c$ is infinite, $c=\{c(0), c(1), \cdots\}$, let

$$
\begin{aligned}
H_{k} & =h_{c(0)}^{-1} \cdots h_{c(k)}^{-1}(M)=h_{c(0)}^{-1} \cdots h_{c(k-1)}^{-1}\left(M_{c(k)}\right), \\
G_{k} & =g_{c(0)}^{-1} \cdots g_{c(k)}^{-1}(M)=g_{c(0)}^{-1} \cdots g_{c(k-1)}^{-1}\left(M_{c(k)}\right),
\end{aligned}
$$

and

$$
r_{k}=g_{c(0)}^{-1} \cdots g_{c(k)}^{-1} h_{c(k)} \cdots h_{c(0)} .
$$

Clearly, $H_{k+1} \subset H_{k}, G_{k+1} \subset G_{k}, G_{k}=r_{k}\left(H_{k}\right)$, and by the lemma, $x=\bigcap_{0}^{\infty} H_{k}$. Furthermore, since $h \sim g$, there are at most an even number of decreasing homeomorphisms in the composition of $r_{k}$, therefore each $r_{k}$ is strictly increasing from the interval $H_{k}$ onto the interval $G_{k}$. Also,

$r_{k}\left(H_{k+1}\right)=g_{c(0)}^{-1} \cdots g_{c(k)}^{-1} h_{c(k)} \cdots h_{c(0)}\left[h_{c(0)}^{-1} \cdots h_{c(k)}^{-1}\left(M_{c(k+1)}\right)\right]=G_{k+1}$.

It follows from these facts that $\bigcap_{0}^{\infty} G_{k}$ is nonempty. To show this we consider three cases.

CASE 1. Each $H_{k}$ is open. Then each $G_{k}$ is open. Let $H_{k}=\left(a_{k}, b_{k}\right)$, $G_{k}=\left(\alpha_{k}, \beta_{k}\right)$. Since $\bigcap_{0}^{\infty} H_{k}$ consists of the point $x$, we must have that $\lim a_{k}=x>a_{k}$ for all $k$ and $\lim b_{k}=x<b_{k}$ for all $k$ (this also means that if $b_{0}=\infty$ some $b_{k}$ must be finite, and similarly, if $a_{0}=-\infty$, some $a_{k}$ is finite). Then there must be infinitely many indices $k$ for which $a_{k}<a_{k+1}$. Let $a_{k}<a<a_{k+1}$. Then $\alpha_{k}<r_{k}(a)<r_{k}\left(a_{k+1}\right)=\alpha_{k+1}$, and therefore if $\alpha=\lim \alpha_{k}, \alpha>\alpha_{k}$ for all $k$. By the same kind of reasoning if $\beta=\lim \beta_{k}, \beta<\beta_{k}$ for all $k$. Since $\alpha \leqq \beta, \cap_{0}^{\infty} G_{k}=[\alpha, \beta]$, nonempty.

CASE 2. Each $H_{k}$ is closed on one end and $k_{0}$ exists such that $H_{k}$ is closed on the same end as $H_{k_{0}}$, say the left for $k \geqq k_{0}$. The $G_{k}$ must have the same property. Let $H_{k}=\left[a_{k}, b_{k}\right), G_{k}=\left[\alpha_{k}, \beta_{k}\right), k \geqq k_{0}$. By the 
same reasoning as in Case 1 if $\beta=\lim \beta_{k}, \beta<\beta_{k}$ for all $k$, therefore $\bigcap_{0}^{\infty} G_{k}=\bigcap_{0}^{\infty}\left[\alpha_{k}, \beta\right]$ which is nonempty.

CAse 3. Each $H_{k}$ is closed on one end but no $k_{0}$ as in Case 2 exists. Then it is easily seen that $\bigcap_{0}^{\infty} G_{k}=\bigcap_{0}^{\infty} \bar{G}_{k}$ which is nonempty. Since in all cases $\bigcap_{0}^{\infty} G_{k}$ is nonempty, there exists $y \in R$ such that $g(y)=c$, which completes the proof.

The proof of Corollary 1 is essentially contained in the analysis of finite sequences given above. Corollary 2 is immediate.

Proof of Theorem 2. Let $h \sim g$ and both be $1-1$ on to $C(h)=C(g)$. Let $x \in R$. The following function $F$ is $1-1$ from $R$ onto $R$ : if $h(x)=c$ then $y=F(x)$ if $g(y)=c$. Since each interval $M_{n}$ consists exactly of those points which correspond under the algorithm to sequences containing at least two entries, the first of which is $n, F$ maps $M_{n}$ onto $M_{n}$. If $h(x)=\{c(0)\}$, then $F(x)=x$ so $F$ maps $I_{n}$ onto $I_{n}$. To see that $F$ is strictly increasing, let $x<\hat{x}, h(x)=c, h(\hat{x})=d$. Define the length $l$ of $c$ as follows: if $c=\{c(0), \cdots, c(k)\}$, then $l=k$, and if $c$ is infinite $l=\infty$. Let $l$ be the length of $d$. There are two cases to consider.

CASE 1. There exists an integer $k \leqq \min (l, \hat{l})$ such that $c(i)=d(i)$, $i<k$, and $c(k) \neq d(k)$. If $k=0$, since $x \in I_{c(0)}, \hat{x} \in I_{d(0)}$, we must have that $I_{c(0)}$ is to the left of $I_{d(0)}$. Since $F(x) \in I_{c(0)}, F(\hat{x}) \in I_{d(0)}, F(x)$ $<F(\hat{x})$. If $k>0$ then we can write

$$
\begin{aligned}
& x=h_{c(0)}^{-1} \cdots h_{c(k-1)}^{-1}\left(x_{k}\right) \quad \text { for some } x_{k} \in I_{c(k)},
\end{aligned}
$$

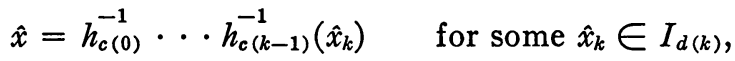

$$
\begin{aligned}
& F(x)=g_{c(0)}^{-1} \cdots g_{c(k-1)}^{-1}\left(y_{k}\right) \quad \text { for some } y_{k} \in I_{c(k)} \text {, } \\
& F(\hat{x})=g_{c(0)}^{-1} \cdots g_{c(k-1)}^{-1}\left(\hat{y}_{k}\right) \quad \text { for some } \hat{y}_{k} \in I_{d(k)} \text {. }
\end{aligned}
$$

Let $h_{c(0)}^{-1} \cdots h_{c(k-1)}^{-1}$ be increasing. Then $x_{k}<\hat{x}_{k}, I_{c(k)}$ is to the left of $I_{d(k)}, y_{k}<\hat{y}_{k}$ and therefore $F(x)<F(\hat{x})$ since $g_{c(0)}^{-1} \cdots g_{c(x-1)}^{-1}$ is also increasing. If $h_{c(0)}^{-1} \cdots h_{c(k-1)}^{-1}$ is decreasing then $x_{k}>\hat{x}_{k}, I_{c(k)}$ is to the right of $I_{d(k)}, y_{k}>\hat{y}_{k}$ and $F(x)<F(\hat{x})$ since $g_{c(0)}^{-1} \cdots g_{c(k-1)}^{-1}$ is also decreasing.

CASE 2. $\min (l, \imath)$ is finite and $c(i)=d(i), i \leqq \min (l, \imath)$. For definiteness let $l<\hat{l}$. If $l=0$ then $x \in I_{c(0)}-M_{c(0)}$. Since $\hat{x} \in M_{c(0)}, x$ is the left end point of $I_{c(0)}$. Then $F(x)=x<F(\hat{x})$ because $F(\hat{x}) \in M_{c(0)}$. If $l>0$ we can write

$$
\begin{array}{rlrl}
x & =h_{c(0)}^{-1} \cdots h_{c(l-1)}^{-1}\left(x_{l}\right), & & x_{l} \in I_{c(l)}-M_{c(l)}, \\
\hat{x} & =h_{c(0)}^{-1} \cdots h_{c(l-1)}^{-1}\left(\hat{x}_{l}\right), & & \hat{x}_{l} \in M_{c(l)}, \\
F(x) & =g_{c(0)}^{-1} \cdots g_{c(l-1)}^{-1}\left(x_{l}\right), & \\
F(\hat{x}) & =g_{c(0)}^{-1} \cdots g_{c(l-1)}^{-1}\left(\hat{y}_{l}\right), & & \hat{y}_{l} \in M_{c(l) .} .
\end{array}
$$


If $h_{c(0)}^{-1} \cdots h_{c(l-1)}^{-1}$ is increasing, $x_{l}<\hat{x}_{l}$, therefore $x_{l}$ is the left endpoint of $I_{c(l)}$, therefore $x_{l}<\hat{y}_{l}$ and $F(x)<F(\hat{x})$. The proof is straightforward if $h_{c(0)}^{-1} \cdots h_{c(l-1)}^{-1}$ is decreasing. Thus $F$ is strictly increasing and is therefore a homeomorphism of $R$ onto $R$. Let $x \in M_{n}$. Then $x=h_{n}^{-1}(r)$ and $F(x)=g_{n}^{-1}(s)$. But $s=F(r)$ (this follows from the fact that if $h(x)=\left\{n, a_{1}, a_{2}, \cdots\right\}$ and $x=h_{n}^{-1}(r)$ then $h(r)$ $\left.=\left\{a_{1}, a_{2}, \cdots\right\}\right)$ therefore $F(x)=g_{n}^{-1}\left(F\left(h_{n}(x)\right)\right)$ or $h_{n}^{-1}=F^{-1} g_{n}^{-1} F$. Conversely let $h_{n}^{-1}=F^{-1} g_{n}^{-1} F$ where $F$ is an increasing homeomorphism from $R$ onto $R$ taking $M_{n}$ onto $M_{n}$. Let $y=F(x)$. Then $h(x)=c$ if and only if $g(y)=c$, which completes the proof.

Proof of Theorem 3 and Theorem 4. Theorem 3 is obtained simply by applying Theorem 2 to this basis, using the inverse functional relation $h_{n}=F^{-1} g_{n} F$ and choosing $g_{n}(x)=1 /(x-n)$ (the corresponding $g$ is the ordinary continued fraction algorithm which is well-known to be 1-1). Theorem 4 is obtained by taking $g_{n}(x)$ $=p \cdot(x-n)$ (the corresponding $g$ is the ordinary decimal expansion to the base $p$, which is 1-1). In both cases the functional relation implies that $F(x)-n$ is a function of $x-n$ only and therefore $F(x)$ $-n=F(x-n)$ for $x \in[n, n+1)$.

Finally, let $\left(A, h_{n}\right)$ be an algorithm basis giving rise to the function $h \in E$ and suppose $h$ is $1-1$. If $x \in R$ and $c$ is an infinite sequence such that $h(x)=c$, there are two ways of interpreting the continued function expansion of $x$ :

$$
x=h_{c(0)}^{-1}\left(h_{c(1)}^{-1}(\cdots)\right) .
$$

The first is that for every $k \geqq 0, x=h_{c(0)}^{-1} \cdots h_{c(k)}^{-1}(y)$ where $h(y)$ $=\{c(k+1), \cdots\}$. The second is that $x=\lim _{k \rightarrow \infty} h_{c(0)}^{-1} \cdots h_{c(k)}^{-1}(y)$ for all $y \in M$, which follows from the fact that $x=\bigcap_{0}^{\infty} H_{k}$.

\section{BIBLIOGRAPHY}

1. B. H. Bissinger, A generalization of continued fractions, Bull. Amer. Math. Soc. vol. 50 (1944) pp. 868-876.

2. C. J. Everett, Representations for real numbers, Bull. Amer. Math. Soc. vol. 52 (1946) pp. 861-869.

3. O. W. Rechard, The representation of real numbers, Proc. Amer. Math. Soc. vol. 1 (1950) pp. 674-681.

4. J. Schreier and S. Ulam, Eine Bemerkung über die Gruppe der topologischen Abbildungen der Kreislinie auf sich selbst, Studia Math. vol. 5 (1934) pp. 155-159.

Los Alamos Scientific Laboratory, University of California, Los Alamos, New Mexico 